\title{
Zellmigration als therapeutischer Zielprozess
}

Ohne die Mobilität von Zellen wäre die normale Entwicklung und Funktion des menschlichen Organismus undenkbar. Aber auch im Rahmen pathologischer Prozesse kommt der Zellwanderung eine Schlüsselrolle zu. Dies hat im Laufe der vergangenen Jahrzehnte in einem translationalen Prozess von bahnbrechenden grundlagenwissenschaftlichen Erkenntnissen über die klassische Sequenz klinischer Studien bis hin zur Zulassung von Medikamenten wie dem anti- $\alpha 4 \beta 7$ Integrin-Antikörper Vedolizumab bei chronisch entzündlichen Darmerkrankungen (CED) geführt $[1,2]$. Darüber hinaus weist das Feld der Zellwanderung insbesondere im Zusammenhang mit gastrointestinalen $\mathrm{Er}$ krankungen allerdings auch bis weit in die Zukunft und lässt neue therapeutische Entwicklungen erhoffen. Aufgrund der zunehmend in den Vordergrund rückenden Erkenntnis, dass die Kommunikation verschiedener Organsysteme mit dem Darm für eine Vielzahl immunvermittelter Erkrankungen von großer Bedeutung ist, eröffnen sich dabei auch völlig neue Perspektiven für darmbezogene Behandlungsansätze bei extraintestinalen Erkrankungen.

Zellwanderung, im englischen Sprachgebrauch „cell trafficking“, beschreibt die Gesamtheit aller passiven und aktiven Bewegungen, die Zellen vollführen [3]. Dazu gehören beispielsweise die amöboide Migration von Zellen in Geweben, daneben allerdings auch Prozesse, die weiträumiger die Bewegung von Immunzellen zwischen verschiedenen Organen steuern. Besonders augenscheinlich lässt sich dies am Beispiel von T-Lymphozyten verdeutlichen, denen bei CED und anderen immunvermittelten entzündlichen Erkrankungen eine pathogenetische Schlüsselrolle zukommt. Nach der Entstehung naiver T-Zellen im Thymus patrouillieren diese auf der Suche nach dem zu ihrem individuellen T-Zell-Rezeptor passenden Antigen durch sekundäre lymphatische Organe. Dabei ermöglicht ihnen die Ausstattung mit Oberflächenmolekülen wie CD62L und CCR7 die Interaktion mit dem Endothel und in den lymphatischen Organen gebildeten Chemokinen, was zu einer gerichteten Auswanderung aus dem Blutstrom führt.
Naive T-Zellen exprimieren außerdem geringe Mengen des Integrins $\alpha 4 \beta 7$, wodurch sie auch in der Lage sind, ins darmassoziierte lymphatische Gewebe (GALT) einzuwandern, auf dessen Endothel der $\alpha 4 \beta 7$-Ligand MAdCAM-1 exprimiert wird.

Wenn eine naive T-Zelle im sekundären lymphatischen Gewebe auf das passende Antigen trifft und entsprechende ko-stimulatorische Signale auf sie einwirken, kommt es zur klonalen Proliferation der dann antigenerfahrenen T-Zelle und in diesem Rahmen zur Differenzierung verschiedener Subpopulationen mit jeweils spezifischer Rezeptorausstattung und assoziierten Besonderheiten des „traffickings“. So werden beispielsweise auf Effektor-TZellen und Effektor-Gedächtnis-T-Zellen CD62L und CCR7 herunterreguliert und stattdessen Oberflächenmoleküle exprimiert, die für den Zugang zum Zielgewebe (in der Regel das Gewebe, das in das Lymphgewebe des Antigenkontakts drainiert) wichtig sind. Im Falle des Darms ist dies insbesondere das Integrin $\alpha 4 \beta 7$. Aufgrund der spezifischen Expression der Retinaldehyddehydrogenase durch dendritische Zellen im Darm wird im GALT aus dem Vitamin A der Nahrung Retinsäure produziert, welche in T-Zellen als Transkriptionsfaktor fungiert und $\alpha 4 \beta 7$-Integrin induziert [4]. So werden die Zellen in die Lage versetzt, nach Rückkehr in den Blutstrom durch Interaktion mit darmspezifisch exprimiertem MAdCAM-1 in den Intestinaltrakt einzuwandern. Während dieser Prozess des Auswanderns aus dem Blutstrom ins Gewebe als „Homing“ bezeichnet wird, existieren weitere aktiv regulierte Prozesse, die die Retention von Immunzellen im Gewebe oder die Rezirkulation zurück ins Blut steuern. Bei der Retention spielen insbesondere die Oberflächenmoleküle CD69 und $\alpha$ E $\beta 7$-Integrin eine Rolle. Letzteres wird im Darmgewebe unter dem Einfluss von TGF- $\beta$ hochreguliert und vermittelt eine Adhäsion an auf dem Epithel exprimiertes E-Cadherin. Ersteres fungiert als Antagonist des ebenfalls in der Zellmembran vorkommenden Sphingosin-1-Phosphat-Rezeptors (S1PR) 1. Durch diese und andere Mechanismen kommt es auch zur Ausbildung einer spezifischen Population von gewebsansässi- gen Gedächtnis-T-Zellen, die im Gewebe verbleibt und dort auf erneuten Antigenkontakt hin „überwacht“ [5]. Die Rezirkulation wird entscheidend durch Sphingosin-1-Phosphat, welches im Blut in hohen Konzentrationen vorliegt und chemotaktisch wirkt, sowie dessen auf T-Zellen exprimierten Rezeptoren vermittelt und ermöglicht eine Rückkehr in den Blutstrom entlang des chemotaktischen Gradienten.

Durch das komplexe Zusammenspiel dieser Mechanismen werden die Menge und der Phänotyp von Immunzellen in peripheren Organen reguliert und so bei Krankheitsprozessen das Entzündungsgeschehen gesteuert. Folgerichtig blockiert der $\alpha 4 \beta 7$-Integrin-Antikörper Vedolizumab das Darmhoming von Immunzellen und wird erfolgreich für die Behandlung von CED eingesetzt.

Darüber hinaus sind mehrere weitere Antikörper und „small molecules“ in der Entwicklung. Für den S1PR-Modulator Ozanimod liegen bereits positive Daten aus Phase-3-Studien [6] und seit Kurzem eine FDA-Zulassung für den Einsatz bei Colitis ulcerosa vor. Mehrere weitere S1PR-Modulatoren werden gegenwärtig in fortgeschrittenen Studienprogrammen evaluiert. Auch der orale $\alpha 4$-Integrin-Agonist AJM 300, der neben $\alpha 4 \beta 7$ auch $\alpha 4 \beta 1$ blockiert, zeigte in einer japanischen Phase3-Studie Wirksamkeit bei Colitis ulcerosa

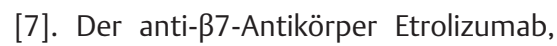
der neben $\alpha 4 \beta 7$ auch $\alpha E \beta 7$ blockiert, konnte zwar in Phase-3-Studien bei Colitis ulcerosa nur begrenzt überzeugen, wird aber bei Morbus Crohn (die Expression von $\alpha E \beta 7$ ist im lleum höher als im Colon) weiter untersucht.

Neben diesen schon weit fortgeschrittenen Entwicklungsprogrammen konnte eine Reihe weiterer molekularer Zielstrukturen wie beispielsweise GPR15 [8] identifiziert werden, deren Modulation zur Beeinflussung des „cell trafficking“ vielversprechend erscheint und bei denen präklinische oder frühe klinische Untersuchungen laufen.

Weit über diese konkreten Ansätze, die im Wesentlichen auf CED fokussieren, hinaus 
können aber auch weitergehende, gegenwärtig noch visionäre Konzepte rund um das „cell trafficking“ bei CED und anderen immunvermittelten entzündlichen Erkrankungen in Betracht gezogen werden. So gibt es überzeugende Evidenz für eine pathogenetische Bedeutung des Darms entlang einer Darm-Gehirn-, Darm-Gelenkoder Darm-Leber-Achse, beispielsweise bei Erkrankungen wie Morbus Parkinson, Rheumatoider Arthritis oder Primär Sklerosierender Cholangitis. Das „trafficking“ von im Darm geprägten Zellen in die jeweiligen Organe scheint hierbei eine wichtige Rolle zu spielen und könnte so neue Behandlungsprinzipien ermöglichen. Zudem erlauben Fortschritte in anderen Disziplinen wie der Biophysik [9] einen völlig neuen Blick auf Wanderungsprozesse und die in diesem Zusammenhang bedeutenden mechanischen Eigenschaften von Zellen, sodass auch hier neue therapeutische Ansätze denkbar sind.

Zusammenfassend kann somit festgestellt werden, dass die pathogenetische Relevanz von „cell trafficking“ und hier ansetzende Therapiestrategien bei CED bereits gut etabliert und weitere Fortschritte in den nächsten Jahren zu erwarten sind. Darüber hinaus scheint es denkbar, dass sich ausgehend von dieser Keimzelle und an der Schnittstelle zu anderen Organsystem und Disziplinen in Zukunft völlig neue Perspektiven auf Zellwanderungsprozesse er- geben, die die Entwicklung gänzlich neuer Therapieoptionen ermöglichen.

\section{Interessenkonflikt}

Der Autor erhielt Forschungsunterstützung von Takeda, Roche und Shire sowie als Advisor und/oder Sprecher Honorare von Takeda, Roche, Janssen, Galapagos, Lilly und Ferring.

Autor

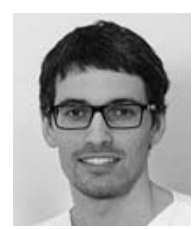

\section{Sebastian Zundler}

Dr. med., Medizinische Klinik 1, Universitätsklinikum Erlangen, Deutschland

\section{Korrespondenzadresse}

Dr. med. Sebastian Zundler

Medizinische Klinik 1

Universitätsklinikum Erlangen

Ulmenweg 18

91054 Erlangen

Deutschland

sebastian.zundler@uk-erlangen.de

\section{Literatur}

[1] Sandborn W], Feagan BG, Rutgeerts $P$ et al. Vedolizumab as induction and maintenance therapy for Crohn's disease. N Engl J Med 2013; 369: 711-721. doi:10.1056/NEJMoa1215739

[2] Feagan BG, Rutgeerts P, Sands BE et al. Vedolizumab as induction and maintenance ther- apy for ulcerative colitis. N Engl J Med 2013; 369: 699-710. doi:10.1056/NEJMoa1215734

[3] Zundler S, Becker E, Schulze LL et al. Immune cell trafficking and retention in inflammatory bowel disease: mechanistic insights and therapeutic advances. Gut 2019; 68: 1688 700. doi:10.1136/gutjnl-2018-317977

[4] Iwata M, Hirakiyama A, Eshima Y et al. Retinoic acid imprints gut-homing specificity on $\mathrm{T}$ cells. Immunity 2004; 21: 527-538. doi:10.1016/j.immuni.2004.08.011

[5] Zundler S, Becker E, Spocinska M et al. Hobitand Blimp-1-driven CD4+ tissue-resident memory T cells control chronic intestinal inflammation. Nat Immunol 2019; 20: 288300. doi:10.1038/s41590-018-0298-5

[6] William S, Geert D, Doug W et al. P025 ozanimod efficacy, safety, and histology in patients with moderate-to-severe ulcerative colitis during induction in the phase 3 True North Study. Official journal of the American College of Gastroenterology I ACG 2020; 115: S6. doi:10.14309/01.ajg.0000722896. 32651.d6

[7] Watanabe M, Matsuoka K, Ohmori T et al. OP34 AJM300, an oral antagonist of $\alpha 4$-integrin, as induction therapy for patients with moderately active ulcerative colitis: a phase 3 , randomized, double-blind, placebo-controlled induction study. Journal of Crohn's and Colitis 2021; 15: S031-S032. doi:10.1093/ecco-jcc/jjab075.033

[8] Kim SV, Xiang WV, Kwak C et al. GPR15-mediated homing controls immune homeostasis in the large intestine mucosa. Science 2013; 340: 1456-1459. doi:10.1126/science. 1237013

[9] Otto O, Rosendahl P, Mietke A et al. Realtime deformability cytometry: on-the-fly cell mechanical phenotyping. Nat Methods 2015; 12: 199-202, $4 \mathrm{p}$ following 202. doi:10.1038/nmeth.3281 